\title{
GPS Remote Sensing Measurements Using Aerosonde UAV
}

\author{
Michael S. Grant ${ }^{*}$ and Stephen J. Katzberg ${ }^{\dagger}$ \\ NASA-Langley Research Center, Hampton, VA, 23681 \\ R. W. Lawrence ${ }^{\ddagger}$ \\ NASA-Langley Research Center, Hampton, VA, 23681
}

\begin{abstract}
In February 2004, a NASA-Langley GPS Remote Sensor (GPSRS) unit was flown on an Aerosonde unmanned aerial vehicle (UAV) from the Wallops Flight Facility (WFF) in Virginia. Using direct and surface-reflected $1.575 \mathrm{GHz}$ coarse acquisition (C/A) coded GPS signals, remote sensing measurements were obtained over land and portions of open water. The strength of the surface-reflected GPS signal is proportional to the amount of moisture in the surface, and is also influenced by surface roughness. Amplitude and other characteristics of the reflected signal allow an estimate of wind speed over open water. In this paper we provide a synopsis of the instrument accommodation requirements, installation procedures, and preliminary results from what is likely the first-ever flight of a GPS remote sensing instrument on a UAV. The correct operation of the GPSRS unit on this flight indicates that Aerosonde-like UAV's can serve as platforms for future GPS remote sensing science missions.
\end{abstract}

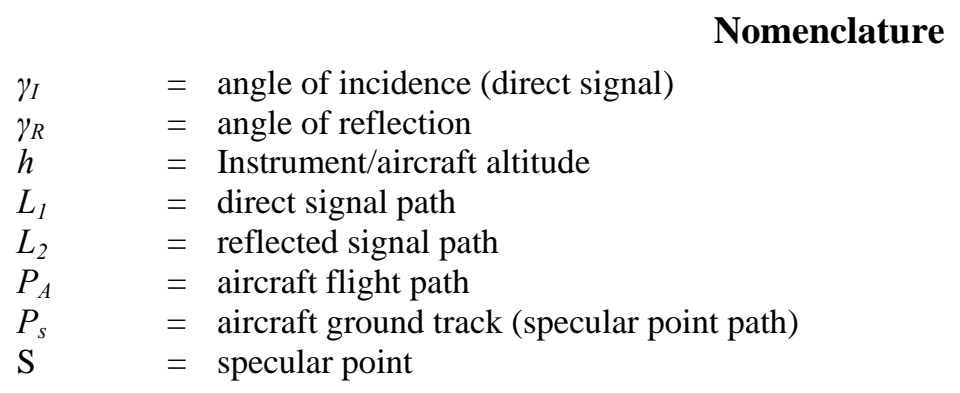

\section{Introduction}

TN recent years the use by NASA of unmanned aerial vehicles (UAV's) for Earth remote sensing has been Lenvisioned. Preliminary efforts are underway to utilize these platforms as substitutes for manned aircraft in order to reduce the cost of field campaigns. NASA's Science Mission Directorate has examined the use of UAV's as suborbital assets and is evaluating their potential in support of global remote sensing missions. In addition, under NASA's Vehicle Systems Program, technology is being developed to enhance the science capability of UAV's. While these technologies will improve vehicle performance, an integrated view of the measurement approach, sensor concept, and vehicle system requirements is necessary to further increase the utility of UAV's for earth and planetary remote sensing.

Given the inherent advantages of UAV's, it is likely that new science measurement capabilities will emerge as innovative remote sensing instruments, targeted specifically for use on UAV platforms, are designed and deployed. New UAV "sensor system" measurements might include ones that require spatial resolutions that are difficult to achieve from low earth orbit (LEO) altitudes, or measurements of extremely localized physical phenomena that require high temporal sampling, e.g. cyclonic storm development or spatial "point sources" such as volcanic eruptions. Based on the initial flight of our GPS remote sensing unit on the Aerosonde UAV, we view as feasible a custom antenna/UAV system designed for long-duration (multi-day or longer) GPS remote sensing of sea state and

\footnotetext{
* Senior Elec. Eng., Systems Engineering Directorate, MS472.

${ }^{\dagger}$ NASA Visiting Professor, South Carolina State University, Orangeburg, SC

₹ Associate Branch Head, Electromagnetics Research Branch, MS490
} 
wind speed over targeted areas. In general, many important and challenging measurements can be obtained or improved by taking an integrated sensor/UAV system approach.

In February 2004, a NASA-Langley GPS Remote Sensor (GPSRS) unit was flown on an Aerosonde UAV from the Wallops Flight Facility (WFF) in Virginia. Using direct and surface-reflected $1.575 \mathrm{GHz}$ coarse acquisition (C/A) coded GPS signals, remote sensing measurements were obtained over land and portions of open water. This was a demonstration flight designed to show that the Aerosonde UAV could accommodate the Langley instrument and that the vehicle could serve as a GPS remote sensing platform. To our knowledge, this was the first-ever flight of a GPS remote sensing instrument on a UAV.

The continuous transmission of L-band signals from GPS satellites means that only a receiving unit is required to obtain measurements. Additionally, the range code inherent in the GPS signal defines the maximum spatial footprint. This allows the use of a smaller, lower gain antenna (6 inch diameter, hemispherical) than what would otherwise be required for similar spatial resolution with a non-range coded signal. The combination of small antenna size, low-weight, and compact electronic packaging makes the Langley GPSRS well suited to science data gathering missions with an Aerosonde-like UAV as the instrument platform.

In this report, we provide a synopsis of the instrument accommodation requirements, installation procedures, and in-flight data acquisition operations for the flight on the Aerosonde. Along with an overview of the GPS remote sensing technique, preliminary wind speed and surface reflectivity results from the flight are presented.

\section{Background}

This section provides background information on the general GPS remote sensing technique as well as on the use of GPS remote sensing for wind speed retrieval over water.

Use of the Global Positioning System (GPS) satellite L-band $(1.57542 \mathrm{GHz})$ signal for remote sensing rather than solely for navigation was begun in the mid-1990's. Katzberg and Garrison performed early theoretical (1996) ${ }^{1}$ and empirical work $(1997)^{2}$ for using the GPS surface-reflected signal to measure ocean wind speeds ${ }^{3,4}$ and to perform remote sensing of wetland areas ${ }^{5}$. Their work included development of the aircraft-based GPSRS instrument (also known as a 'Delay Mapping Receiver'), which is capable of simultaneously measuring both the direct and reflected signals from one or more GPS satellites. Since the GPS signal is strongly reflected from water, studies by Masters, Zavorotny, Katzberg, and others ${ }^{6-8}$ have recently focused on characterization of the landreflected GPS signal as a function of soil moisture. Previous work in this area also includes our use of GPS landreflected signals in combination with traditional aerial imagery for unsupervised terrain feature classification ${ }^{9}$.

\section{A. GPS Remote Sensing Technique}

In this study, both the directly transmitted, right-hand circularly polarized (RHCP) satellite signal and the surface-reflected, left-hand circularly polarized (LHCP) signal are used. Signals (direct and reflected) are obtained from the highest satellite in view, and are acquired using RHCP and LHCP antennas on the top and underside, respectively, of the aircraft. The calibrated ratio of reflected-to-direct instrument output signals provides a measure of surface reflectivity. Additionally, the unique satellite identification code embedded in the GPS signal, along with known GPS satellite geometry and local elevation data, allows the GPS signal mirror reflection (specular) point ground track to be calculated and plotted on a geographic coordinate grid.

Fundamentally, the strength of the surface-reflected GPS signal is proportional to the amount of moisture in the surface and is also influenced by surface roughness. Because the signal is strongly reflected from surface water, the use of amplitude and other characteristics of the reflected signal allow an estimate of wind speed over open water.

Figure 1 shows the direct and surface-reflected GPS signal paths from the satellite to the GPSRS unit. Signal path $L 1$ is the direct path from the satellite to the airborne instrument located $h$ meters $(\mathrm{m})$ above local terrain. Path $L 2$ is from the satellite to the $x-y$ surface plane of the terrain, and then from the surface to the instrument (dotted line). To compute specular point, $S$, the surface is assumed to be flat and mirror-like to the GPS signal. Thus the angle of reflection, $\gamma_{R}$, equals the incident angle, $\gamma_{I}$. Note that the shortest reflected signal path is through point $\mathrm{S}$. For the near-specular case, only signals reflected from a region of the surface approximately the size of the first Fresnel zone (centered at point $S$ ) will reach the LHCP antenna.

As the airborne instrument moves along path $P_{A}$, parallel to the x-y surface, the specular point ground track (or simply the "ground track"), $P_{S}$, is formed along the surface. Note that GPS only provides altitude measurements relative to the WGS84 ellipsoid model of the earth's surface ${ }^{10}$, therefore local terrain elevation data is required to compute the true altitude, $h$. 
In operation, the GPSRS records data values proportional to the direct and reflected signal power at 0.1-second intervals. The direct signal, which has a relatively constant power level, is used to obtain aircraft position. Reflected signal power is concentrated around specular point $S$, and decreases with distance from the specular point (and with the corresponding increase in signal transit time delay). This power vs. [time] delay characteristic of the reflected signal is measured by the instrument and is used for wind speed estimation.

\section{B. Wind speed Measurement Technique}

An outline of the wind speed measurement technique is presented here, with further detail given in Ref. 11.

From signal theory, it is known that the maximum response of a linear [time-invariant] system is obtained when the system impulse response is a time-reversed copy of the input waveform ${ }^{12}$. As discussed in Ref. 12, such a "matched filter" system will produce the optimum signal-to-noise ratio given an input signal contaminated only by Gaussian white noise. In this instance, the time-reversed impulse response results in the system output convolution becoming a crosscorrelation.

For the GPS case, the signal is contaminated with additive thermal noise not correlated with the transmitted signal, and we also note that the transmitted signal power at

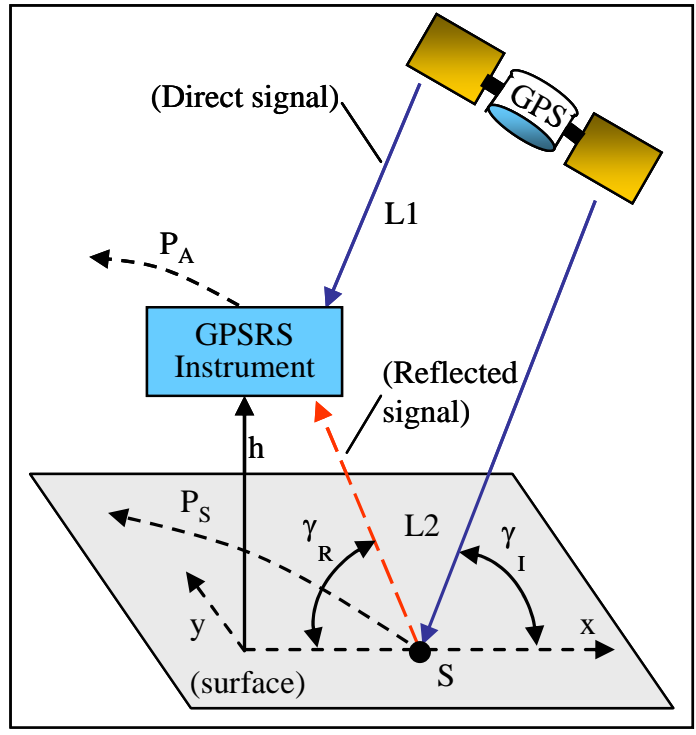

Figure 1. GPS remote sensing geometry. Direct (L1) and Reflected (L2) Signal Paths to GPS Remote Sensor (GPSRS). the earth's surface is actually well below the additive thermal noise power of the receiver front-end ${ }^{13}$. When the transmitted signal encounters the surface of rough water, what is often termed "fading noise" results due to the random phase shifts associated with individual wave facets, i.e. individual portions of the reflecting surface. The total noise in the reflected signal is therefore comprised of fading noise plus additive thermal noise from the receiver.

In the data processing software, the matched filter method is implemented by taking the correlation of the received power vs. delay waveform with a number of possible template waveforms, given the aircraft altitude and satellite elevation angle. Wind speed is then inferred from the ideal waveform that results in the maximum correlation. Even with a theoretically non-ideal noise condition, because of the relative stability of the transmitted GPS signal power during nominal flight times (on the order of 30 minutes to two hours) and subsequent stable noise characteristics, the matched filter technique has proven effective for measuring both land and water reflections.

\section{Aerosonde Overview and Instrument Accommodation}

A brief overview of the Aerosonde UAV is provided in this section, along with GPS instrument accommodation requirements for the flight.

The Aerosonde is a single-engine unmanned aerial vehicle with a 10-foot wingspan, weighing $27-30$ pounds [13]. The UAV can be remotely piloted or may be operated in an autonomous mode. For this flight, the UAV was remotely piloted by a trained Aerosonde operator. In addition to onboard communication and navigation electronics, the vehicle can carry an additional five-pound payload with a full fuel load.

The GPS unit used in this study weighs approximately four pounds and consists of a multi-layer stack of electronic boards in the PC/104 form factor. During operation the unit draws approximately 500mA at a nominal 18 volts (V), and can operate with a supply voltage of between 12 and $40 \mathrm{~V}$. The board stack was placed directly in the Aerosonde fuselage and the unit was powered with $18 \mathrm{~V}$ supplied by a generator onboard the UAV. No enclosure for the remote sensor unit was used for this flight although for future missions a shielded electronics enclosure, customized to Aerosonde power connections and fuselage dimensions, would likely be built.

Once power was applied to the UAV, the GPS unit began acquiring data and recording the location of the vehicle from launch through landing (Fig. 2). All GPS data (both direct and reflected channel data) were stored in non-volatile ("Flash") memory and were processed post-flight. For future long-duration missions, planned enhancements to the GPS receiver software will allow data to be downlinked via the Iridium satellite system for near real-time processing and data display. 


\section{Measurement Results}

Figure 2 shows the Aerosonde flight track and color-coded reflected signal data superimposed on a digital orthoquad image (georeferenced pixels) of the Wallops Flight Facility area. As can be seen in the figure, the vehicle flew over land, marsh, man-made facilities and open water. Because of the aircraft agility in flight, sharp turns sometimes resulted in loss of synchronization with the satellite signal. Evidence of signal loss may be seen from the discontinuities in the ground tracks of Figure 2.

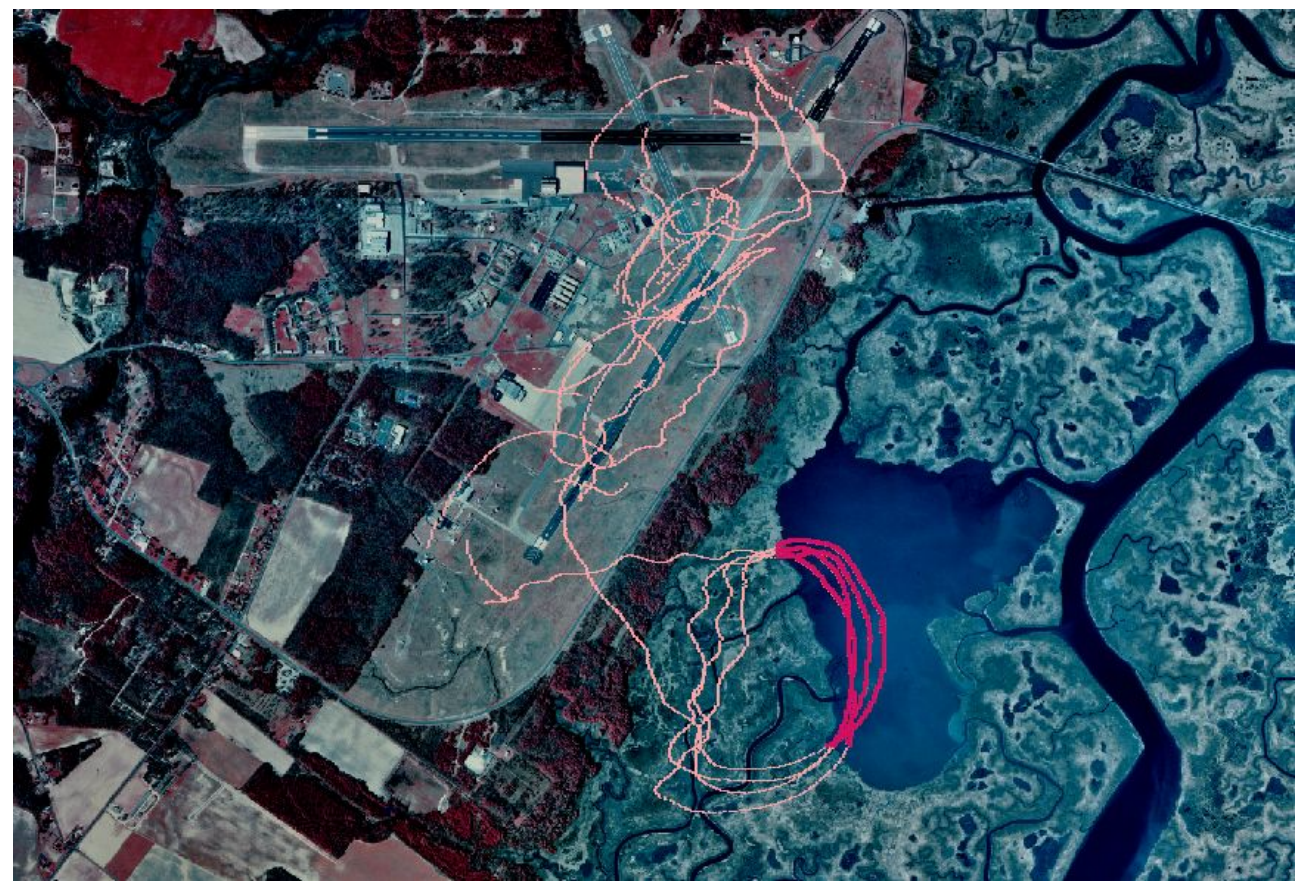

Figure 2. Aerosonde ground track and signal.

Color-coded reflected signal data/ground track of Aerosonde flight, on 27 Feb. 2004, over Wallops Flight Facility near Chincoteague, Virginia (tracks on orthoquad image). Darker red indicates stronger signal.

Wind speed retrievals were performed using portions of the flight data over open water (Figure 3). These segments of the flight path coincide with a tidal marsh lagoon. Because the image in Figures 2 and 3 is from 1992, it is possible that some migration of the lagoon may have occurred between 1992 and the flight in 2004. However major changes in the landscape do not appear to have occurred, given that the data (dark red color-coded data) match the boundaries of the lagoon relatively well.

The GPS-derived wind speed $(\mathrm{m} / \mathrm{s})$ is plotted in Figure 4 as a function of flight path latitude, at 0.1 second intervals. The retrieved wind speed varies from approximately 3 to $15 \mathrm{~m} / \mathrm{s}$, and is approximately $11 \mathrm{~m} / \mathrm{s}$, on average. Buoy and weather station data (Delaware Bay buoy) indicate that wind speeds in the area during the flight were fairly steady at $11.8 \mathrm{~m} / \mathrm{s}$ (with gusts to $13.8 \mathrm{~m} / \mathrm{s}$ ) and $11.3 \mathrm{~m} / \mathrm{s}$ (with gusts to $13.2 \mathrm{~m} / \mathrm{s}$ ) at 1400 hours and 1500 hours, respectively (Table 1 ).

Although this flight of the GPS system was primarily for demonstration purposes and not for quantitative results, the GPS-derived wind speeds are reasonable when compared to the buoy data. During typical wind speed measurement missions, data are generally acquired over larger portions of open water, uninterrupted by land, as compared to the data runs on this initial UAV flight. Given the fairly constant wind speeds from the buoy, measurements over a larger body of water would likely have had lower variability than that observed during this flight. 


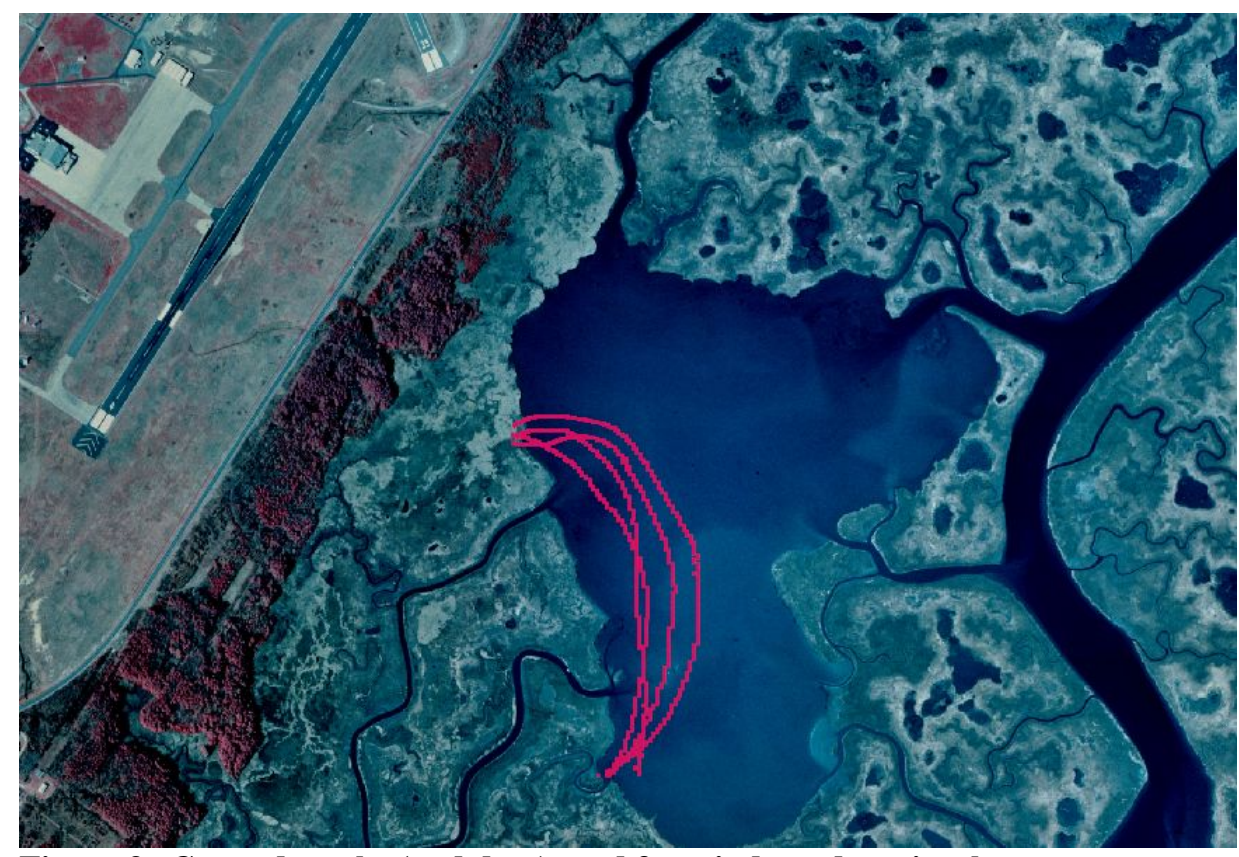

Figure 3. Ground tracks (and data) used for windspeed retrieval

Ground tracks over marsh lagoon showing location of data used for wind speed retrieval.

Table 1. Delaware Bay Buoy 44009 February 27, 2004, 1400 and 1500 hours.

\begin{tabular}{|c|c|c|c|}
\hline Hour & $\begin{array}{c}\text { Direction } \\
\text { (degrees) }\end{array}$ & $\begin{array}{c}\text { Wind Speed } \\
(\mathrm{m} / \mathrm{s})\end{array}$ & $\begin{array}{c}\text { Gust Speed } \\
(\mathrm{m} / \mathrm{s})\end{array}$ \\
\hline 1400 & 30 & 11.8 & 13.7 \\
\hline 1500 & 32 & 11.3 & 13.5 \\
\hline
\end{tabular}

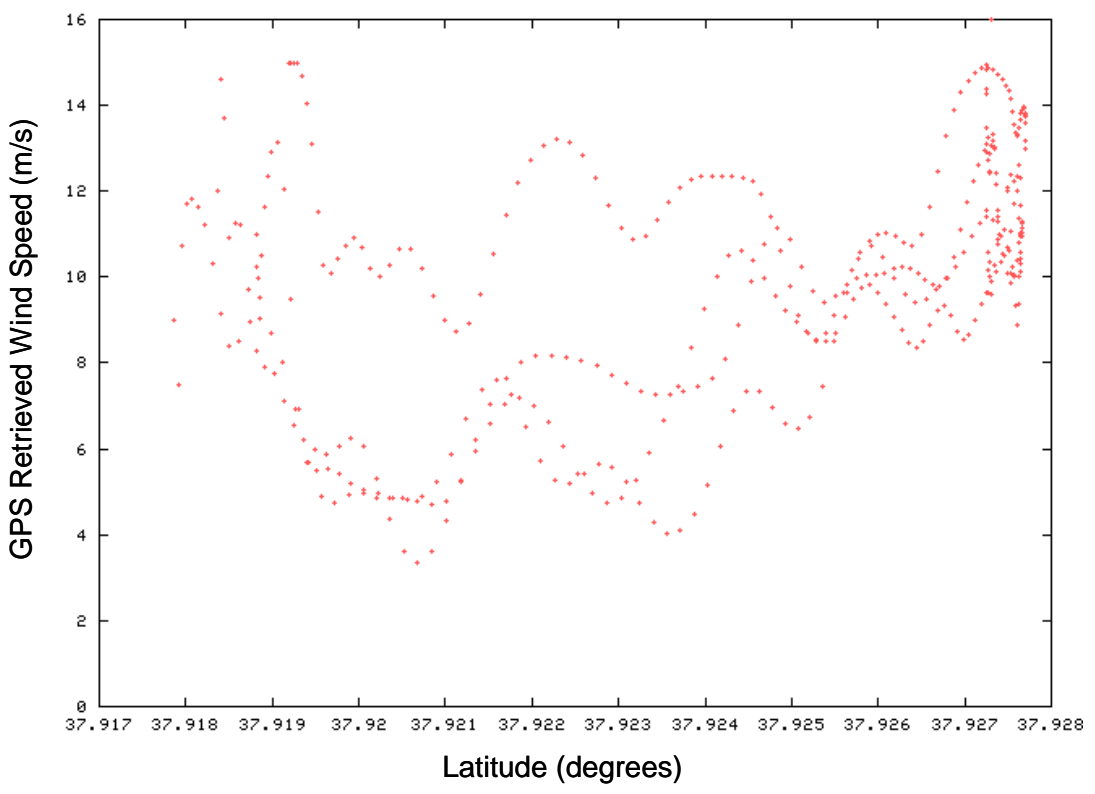

Figure 4. GPS-Measured Wind Speed. Wind speed in meters per second vs. latitude (degrees) of the flight path over the marsh lagoon. 
Figure 5 is a plot of the preliminary (uncalibrated) surface reflectivity, i.e. the reflected-to-direct channel ratio. We note that there was a significant fluctuation in the direct signal that depended on aircraft flight direction. Although this "multipath" reflection effect has been seen on a number of previous missions, the signal variation may have been accentuated by the lack of a metallic ground plane for the top (direct) antenna. As mentioned previously, the combined antenna and vehicle configuration would be a key part of any dedicated UAV/GPS remote sensor system design.

In order to compute surface reflectivity, the reflected channel values (digital counts) were divided by the average value of the direct channel. Due to temporary losses of satellite lock during the flight, further processing would be required to calibrate and fully align the data with surface features. This being the case, the uncalibrated surface reflectivity is still in a range ( $\sim 0$ to 1.5$)$ that has been observed on previous missions. This, along with reasonable windspeed measurements, indicates that the instrument was functioning properly.

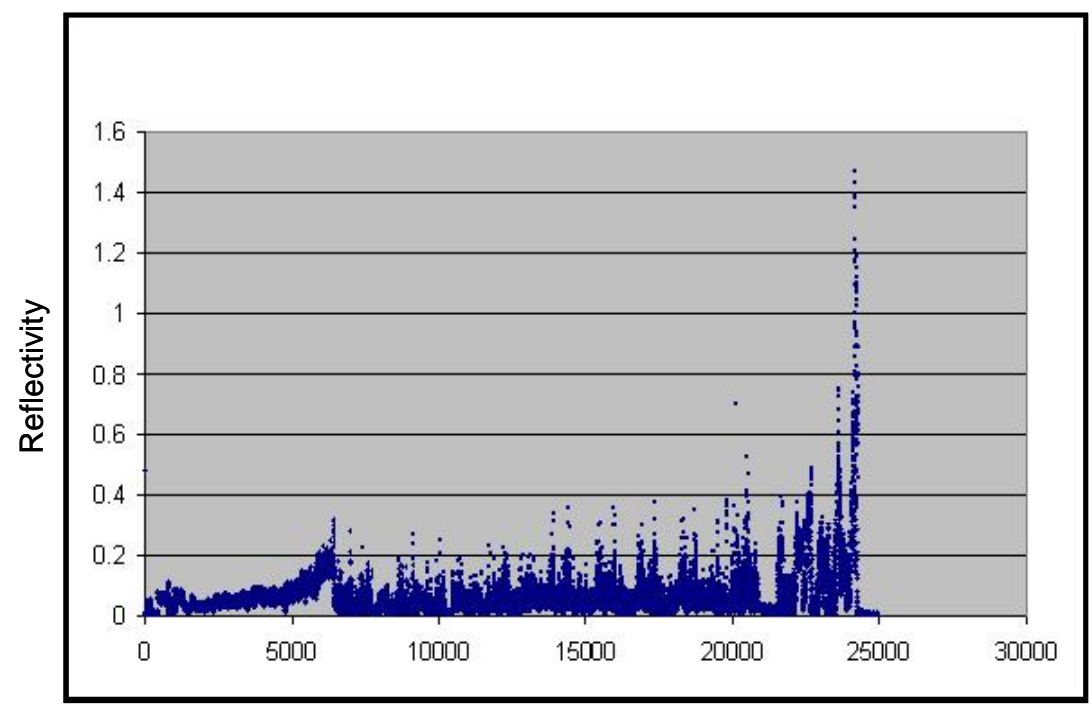

Sample number

Figure 5. GPS Reflectivity. Preliminary (uncalibrated) reflectivity, reflected-to-direct channel ratio.

\section{Conclusion}

In this paper, an overview of what is, to our knowledge, the first-ever flight of a GPS remote sensing instrument on an unmanned aerial vehicle has been presented. The utility of an Aerosonde-like vehicle for use as a GPS remote sensing platform has been demonstrated by successful operation of the Langley GPSRS instrument on the Aerosonde UAV. Although designed as a demonstration flight, data were acquired that allowed a relatively accurate determination of wind speed, as compared to buoy data. Additionally, the uncalibrated surface reflectivity was consistent with reflectivities measured on previous flights over areas of dry land and water.

For future missions, the antenna ground plane and/or electromagnetic shielding will likely need to be improved in order to reduce the variability of the direct signal. To avoid temporary loss of satellite lock, the UAV pilot must minimize the steepness of aircraft turns, especially over targeted measurements areas. The Aerosonde or similar UAV's that are remotely piloted or that operate autonomously, and which can remain on-station for relatively long periods, clearly show promise as GPS remote sensor data gathering platforms. 


\section{References}

${ }^{1}$ Katzberg, S. J., and Garrison, J. L., “Utilizing GPS to Determine Ionospheric Delay Over the Ocean,” NASA TM-4750, 1996.

${ }^{2}$ Garrison, J. L., Katzberg, S. J., and Howell, C. T.,III, "Detection of Ocean Reflected GPS Signals: Theory and Experiment,” Proceedings of the IEEE SoutheastCon 1997 Conference, Blacksburg, VA, 1997, pp. 290-294.

${ }^{3}$ Garrison, J. L, Katzberg, S. J., and Hill, M. I., "Effect of Sea State Roughness on Bistatically Scattered Range Code Signals from the Global Positioning System,” Geophys. Research Letters., Vol. 25, 2257-2260, 1998.

${ }^{4}$ Garrison, J. L., Katzberg, S. J., Zavorotny, V. U., and Masters, D., "Comparison of Sea Surface Wind Speed Estimates From Reflected GPS Signal With Buoy Measurements,” Proceedings of the IEEE Geoscience and Remote Sensing Symposium (IGARSS), Vol. 7, Honolulu, HI, 2000, pp. 3087-3089.

${ }^{5}$ Garrison, J. L. and Katzberg, S. J., “The Application of Reflected GPS Signals to Ocean and Wetland Remote Sensing," Proceedings of the 5th Intl. Conf. on Remote Sensing for Marine and Coastal Environments Conference, Vol. 1, San Diego, CA, 1998, pp. 522-529.

${ }^{6}$ Masters, D, Zavorotny, V. U., Katzberg, S. J., and Emery, W., "GPS Signal Scattering from Land for Moisture Content Determination,” Proceedings of the IEEE Intl. Geoscience and Remote Sensing Symposium (IGARSS), Vol 7., Honolulu, HI, 2000, pp. 3090-3092.

${ }^{7}$ Masters, D, Katzberg, S. J., and Axelrad, P., “Airborne GPS Bistatic Radar Soil Moisture Measurements During SMEX02,” Proceedings of the IEEE Intl. Geoscience and Remote Sensing Symposium (IGARSS), Vol. 2, Toulouse, France, 2003, pp. 896 898.

${ }^{8}$ Katzberg, S. J., Torres, O., Grant, M. S., and Masters, D., "Utilizing Calibrated GPS Reflected Signals to Estimate Soil Reflectivity and Dielectric Constant: Results From SMEX02,” Remote Sensing of Environment (submitted for publication, June 2005).

${ }^{9}$ Grant, M. S. and Katzberg, S. J., “Combined GPS Reflected Signal and Visual Imagery for Unsupervised Clustering and Terrain Classification,” Proceedings of the IEEE SoutheastCon 2004 Conference, Vol. 1, Greensboro, NC, 2004, pp. 370-377.

${ }^{10}$ B. Hofmann-Wellenhof, B., Lichtenegger, H., and Collins, J., GPS Theory and Practice, $5^{\text {th }}$ ed., Springer-Verlag Wien, New York, 2001.

${ }^{11}$ Garrison, J. L. and Katzberg, S. J., "Comparison of Sea Surface Wind Speed Estimates From Reflected GPS Signals With Buoy Measurements,” Proceedings of the IEEE Intl. Geoscience and Remote Sensing Symposium (IGARSS), Vol. 7, Honolulu, HI, 2000, pp. 3087-3089.

${ }^{12}$ Carlson, A. B., Communication Systems: An Introduction to Signals and Noise in Electrical Communication, McGraw-Hill, New York, 1968, Chaps. 3, 9.

${ }^{13}$ Parkinson, B. W., Spilker, J. J. (editors), Global Positioning System: Theory and Applications, Vol. 1, AIAA, Washington, DC, 1996, pp. 84-86.

${ }^{14}$ Aerosonde Pty. Ltd. (AePL), Aerosonde System Overview document, Mark 3 UAV, Table 1, p. 1, URL: http://www.aerosonde.com/drawarticle/26 [cited 9 August 2005]. 